\title{
Role of Mitochondria in Parkinson Disease
}

\author{
Siegfried Kösel ${ }^{1}$, Götz Hofhaus ${ }^{2}$, \\ Alexander Maassen ${ }^{2}$, Peter Vieregge ${ }^{3}$ and \\ Manuel B. Graeber ${ }^{4, *}$ \\ ${ }^{1}$ Labor für Molekulare Neuropathologie, Institut \\ für Neuropathologie, Ludwig-Maximilians-Universität \\ München, Thalkirchner Str. 37, D-80337 München, \\ Germany \\ ${ }^{2}$ Institut für Biochemie, Heinrich-Heine-Universität \\ Düsseldorf, Universitätsstr. 1, D-40225 Düsseldorf, \\ Germany \\ ${ }^{3}$ Klinik für Neurologie, Medizinische Universität Lübeck, \\ Ratzeburger Allee 160, D-23538 Lübeck, Germany \\ ${ }^{4}$ Labor für Molekulare Neuropathologie, \\ Abteilung für Neuromorphologie, Max-P lanck-Institut \\ für Neurobiologie, Am Klopferspitz 18a, \\ D-82152 M artinsried, Germany \\ *Corresponding author
}

The cause of the selective degeneration of nigrostriatal neurons in Parkinson disease (PD) has remained largely unknown. Exceptions include rare missense mutations in the alpha-synuclein gene on chromosome 4 , a potentially pathogenic mutation affecting the ubiquitin pathway, and mutations in the parkin gene on chromosome 6. However, unlike classical PD, the latter syndrome is not associated with the formation of typical Lewy bodies. In contrast, a biochemical defect of complex I of the mitochondrial respiratory chain has been described in a relatively large group of confirmed PD cases. Recent cybrid studies indicate that the complex I defectin PD has a genetic cause and that it may arise from mutations in the mitochondrial DNA. Sequence analysis of the mitochondrial genome supports the view that mitochondrial point mutations are involved in PD pathogenesis. However, although mitochondria function as regulators in several known forms of cell death, their exact involvement in PD has remained unresolved. This is of relevance because classical apoptosis does not appear to play a major role in the degeneration of the parkinsonian nigra.

Key words: Apoptosis / Complex I / Mitochondrial DNA / Neurodegeneration.

\section{Introduction}

Parkinson disease (PD) is a common neurological disorder that affects more than $2 \%$ of the population older than 65 years. The disease is clinically characterized by a combination of the four card inal symptoms, bradykinesia, rigidity, resting tremor and loss of postural reflexes. In addition, affected individuals may be cognitively impaired. Due to the significant overlap of clinical symptoms between PD and several other neurodegenerative disorders, the definitive diagnosis still rests on careful examination of the brain and spinal cord post mortem. The most striking neuropathological findings in PD include depigmentation of the Substantia nigra, which results from a loss of pigmented dopaminergic neurons, and the presence of Lewy bodies in surviving nerve cells. Lewy bodies are also found in related nuclei of the brain stem such as the Locus coeruleus. By definition, Lewy bodies are a conditio sine qua non for a definitive diagnosis ofPD. They are bestseen in hematoxylin-eosin stained tissue sections and are immunoreactive for a number of proteins, including neurofilaments, ubiquitin and alpha-synuclein (vide infra). Their role in PD is still unclear.

Evidence is accumulating that the clinico-pathological 'entity' PD in fact represents an etiologically heterogeneous group of disorders. Genetic as well as environmental factors, including oxidative cell damage and accelerated aging, are likely to contribute to the multifactorial etiology of this complex disease. Familial forms of PD are rather uncommon (10-15\% of the cases) but rare missense mutations have been identified in the alpha-synuclein gene on chromosome 4 (Polymeropoulos et al., 1997; Krüger et al., 1998). Although the frequency of alpha-synuclein mutations is extremely low, this protein now represents the most sensitive marker of Lewy bodies also in idiopathic cases of the disease. Thus, alpha-synuclein is likely to have a central position in the pathway the function of which is disturbed in PD. In addition, a potentially pathogenic mutation affecting the ubiquitin pathway (Leroy et al., 1998) and mutations in the parkin gene on chromosome 6 (Kitada et al., 1998; Lücking et al., 1998) have been described in some families. However, unlike classical PD, the latter syndrome is not associated with the formation of typical Lewy bodies. As indicated above, the overwhelming majority of PD cases is sporadic and lacks a family history making molecular genetic analyses difficult. Availability of sufficient numbers of appropriate controls for genetic studies, which need to be matched not only for age and sex but also for ethnic background, represents another problem. As a result, the finding of associations between several nuclear candidate genes and $P D$ has remained controversial. 


\section{Mitochondrial Dysfunction in Parkinson Disease}

First hints pointing to a contribution of mitochondria to PD pathogenesis were obtained on the basis of a study by Langston and colleagues in 1983 (Langston et al., 1983). These authors observed that the neurotoxin MPTP (1methyl-4-phenyl-1, 2, 3, 6-tetrahydropyridine) causes a PD-like syndrome in humans. The toxin acts via inhibition of complex I of the mitochondrial respiratory chain (McNaught et al., 1996). Subsequently, several studies reported on a decrease of complex I activity in the Substantia nigra of PD brains (Schapira et al., 1989, 1990; M izuno et al., 1989; J anetzky et al., 1994). In some patients, a decrease of complex I activity was also observed in platelets (Krige et al., 1992; Benecke et al., 1993) and skeletal muscle (Bind off et al., 1991).

Complex I of the mitochondrial respiratory chain is localized at the inner mitochondrial membrane and forms part of the oxidative phosphorylation (OXPHOS) system of the mitochondrion, which is responsible for the production of cellular ATP. The OXPHOS system consists of four large enzyme complexes (complexes I, III, IV and V). Here, electrons flow starting from $\mathrm{NADH}$ and $\mathrm{FADH}_{2}$ through complexes I, III, and IV (respiratory chain) and are finally transferred to $\mathrm{O}_{2}$ which is oxidized to $\mathrm{H}_{2} \mathrm{O}$. The energy released during oxidation is conserved by the vectorial transport of protons from the mitochondrial matrix into the intermembrane space. The resulting 'chemiosmotic gradient' is then used by complex V, or ATPase, for the synthesis of ATP (Figure 1).

Complex I encompasses more than 40 protein subunits with their majority being encoded by the nucleus. However, the exact genomic location of most of these genes is still unknown (Duncan et al., 1992; Kim et al., 1997; Russel et al., 1997). In contrast, the genetic information for seven polypeptide chains of mitochondrial complex I, termed ND1, ND2, ND3, ND4, ND4L, ND5, and ND6, is encoded by the mitochondrial DNA (mtDNA) (Figure 1). The entire sequence of the mitochondrial genome has been known for almost 20 years (Anderson et al., 1981). This allows detailed mutation analyses. mtDNA represents a doublestranded, circular molecule of $16569 \mathrm{bp}$ in length. There are several copies of the mitochondrial genome per organelle and several hundred mtDNA molecules per cell. mtDNA codes for a total of 13 proteins which all are components of the OXPHOS system in addition to two rRNA molecules and 22 mitochond rial tRNAs (Wallace, 1992).

There are several important differences between mitochondrial and nuclear genetics. mtDNA is transmitted exclusively through the maternal line. Furthermore, the mutation rate of mtDNA is approximately 10-20 times higher than that of nuclear DNA. Reasons for this include an insufficiency of the mitochondrial DNA repair system, lack of protective histone-like proteins and the physical proximity of the mitochond rial chromosome to the OXPHOS system which generates oxygen radicals even in its normal state. Due to the high copy number of the mtDNA, varying amounts of normal and mutated mtDNA species can coexist in the same cell. This state is referred to as heteroplasmy. During cell division in mitotically active tissues, mitochondria segregate randomly into daughter cells. This will create differences in the proportion of mutant mtDNA between different cells and tissues. Thus, in the case of a heteroplasmic mtDNA mutation, expression of its clinical phenotype may depend on tissue specific thresholds. Therefore, mitochond rial mutations are ideally studied in the anatomical region of their postulated pathogenic effect, i. e., the Substantia nigra in PD.

Recent studies indicate that there is, in fact, a contribution of mtDNA to PD pathogenesis. Studies on PD families have demonstrated a matrilineal pattern of inheritance in some instances (Wooten et al., 1997; Swerdlow et al., 1998). In addition, two ind ependent groups using mtDNAless human $\rho^{0}$ cells repopulated with mitochondria derived from platelets of PD patients and controls (Swerdlow et al., 1996; Gu et al., 1998; Figure 2) were able to show that the parkinsonian complex I defect is transmittable by mtDNA. These same authors reported a mean decrease of $20-25 \%$ in complex I activity and increased oxygen radical production in PD cybrid cells compared with controls. Moreover, studying PD families, cybrid cell lines containing mtDNA from maternal descendants showed lower complex I activity, increased oxygen radical formation and morphologically abnormal mitochondria (Swerdlow et al., 1996). Corresponding results were also obtained on sam-

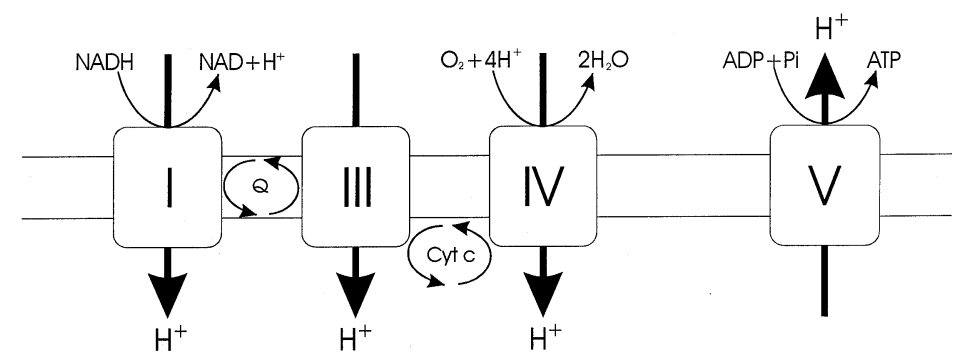

Origin of Subunits

$\begin{array}{rrrrr}\text { MtDNA } & 7 & 1 & 3 & 2 \\ \text { Nuclear DNA } & >33 & 10 & 10 & 10\end{array}$

Fig. 1 Schematic Drawing of the Mitochondrial Respiratory Chain and Oxidative Phosphorylation System (OXPHOS). 

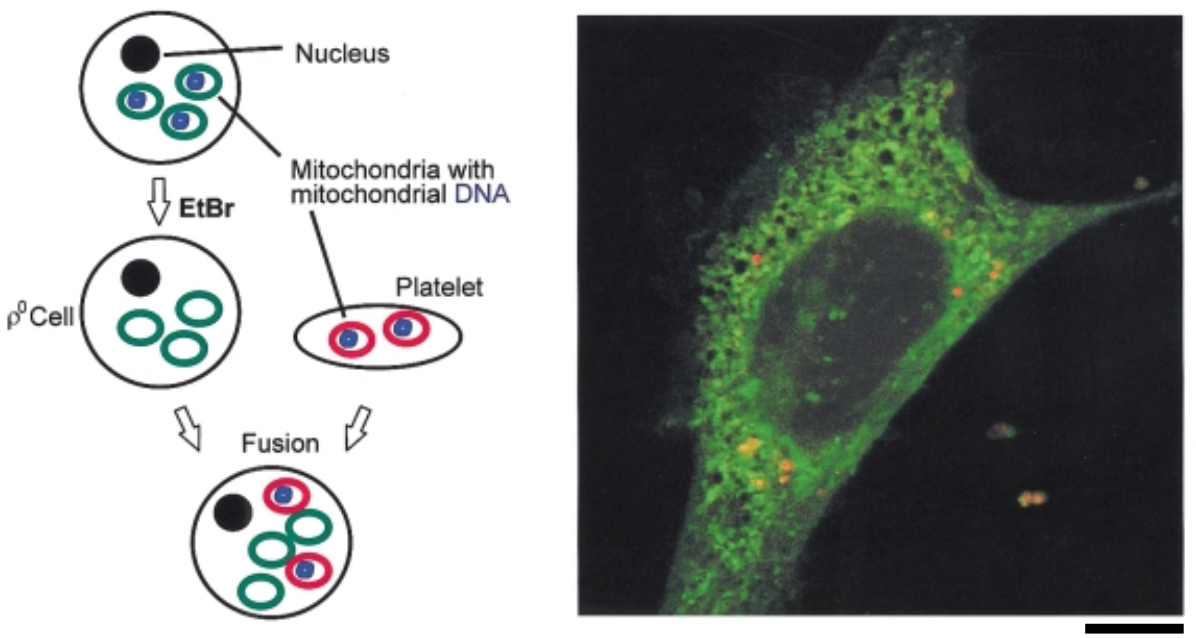

Fig. 2 Generation of Cybrids using $\rho^{0}$ Cells (Which Lack mtDNA) and P latelets of PD Patients Who Carry mtDNA Point Mutations to Study Functional Aspects of these Mutations.

(A) $\rho^{0}$ cells are generated by incubation with ethidium bromide ( $\mathrm{EtB} \mathrm{r}$ ). EtB r removes $\mathrm{mtDNA}$ from the cells which are then cultured in an uridine-supplemented medium. Following the fusion of $\rho^{0}$ cells with the patients' platelets, the mitochond rial functional phenotype of the patients is expressed by the cybrid cell line. (B)Confocal image of a cybrid cell. Platelet mitochondria and mitochondria of the $\rho^{0}$ cell were labeled with MitoTracker red and green, respectively. Scale bar: $15 \mu \mathrm{m}$.

ples from symptomatic PD patients and asymptomatic young maternal siblings (Swerdlow et al., 1998). In contrast, no mitochondrial transmission of the complex I defect was found in cybrids generated with platelets from patients affected by dystonia (Tabrizi et al., 1998). Thus, the mitochond rial complex I defect in PD is likely to be due to an mtDNA abnormality in a subset of PD patients. Its postulated mechanism of action is an elevated production of oxygen radicals leading to an increased susceptibility of nigra neurons to cell death. The PD Substantia nigra is especially sensitive to oxygen radical damage because its level of glutathione is reduced (Sian et al., 1994). In addition, the neurotransmitter metabolism of dopaminergic neurons produces reactive oxygen species even under normal conditions. The toxicity of the latter may be further increased by the large amounts of iron which are present in the parkinsonian Substantia nigra (Hartley et al., 1993).

\section{Molecular Genetic Analysis of mtDNA in Parkinson Disease}

High throughput analysis of the mitochondrial genome in $P D$ is in its infancy. Efforts to elucidate the genetic basis of the parkinsonian complex I defect by systematic sequencing of the mitochondrial genome have so far been confined to little more than 30 cases worldwide. Early studies employing Southern blotting had failed to detect deletions of the mtDNA genome in PD (Lestienne et al., 1990). Great care has to be taken when interpreting individual studies because not all groups have used DNA isolated from the Substantia nigra and, more importantly, from neuropathologically verified cases. In addition, there is firm evidence now that the frequency of certain mtDNA sequence variants may vary greatly depending on ethnic background.

The so-called 'common deletion' of mtDNA which spans $4977 \mathrm{bp}$ and which removes appx. $40 \%$ of complex I genes has been implicated in PD. The concentration of this mtDNA species has been suggested to increase in the parkinsonian nigra and to be responsible for the observed biochemical defect of complex I (Ozawa et al., 1990). However, we and others (Mann et al., 1992; Kösel et al., 1997a) have been unable to confirm this finding and rather believe that the overall moderate increase ( $5 \%$ vs. control) in the amount of mtDNA carrying the 'common deletion' probably represents an age-related phenomenon.

Ikebe and coworkers sequenced DNA which had been extracted from the striatum of five PD brains. Although there were no specific point mutations among the patients in contrast to several neuromuscular disease cases, they found several point mutations in each brain which should result in a significant change of the gene product. They concluded that some of the mutations may be risk factors for PD which increase the susceptibility of nigra neurons to oxidative damage (Ikebe et al., 1995). The Wallace group (Brown et al., 1996) has completely sequenced mtDNA from four patients three of whom, however, exhibited neuropathological changes of PD and Alzheimer disease at the same time. The single 'PD only' patient carried two novel sequence variants. One was detected at nucleotide position 15851 of the cytochrome b (cytb) gene and another one at position 1709 of the gene encoding the 16S rRNA. The 'PD + Alzheimer' patients also harbored mtDNA sequence changes which were considered likely to contribute to the observed neuropathology (Brown et al., 1996). Interestingly, one of these patients carried the mtDNA $4336 \mathrm{~A} / \mathrm{G}$ mutation which affects the mitochondrial tRNA for glutamine. Other studies have shown that 
this sequence variant represents a risk factor for both $P D$ and Alzheimer disease (Shoffner et al., 1993; Egensperger et al., 1997).

To further clarify the contribution of mitochondrial sequence variation to PD pathogenesis we have sequenced all mitochond rial complex I genes as well as the mitochondrial tR NA genes in 22 (20) cases of neuropathologically confirmed PD (Kösel et al., 1998; Grasbon-Frodl et al., 1999). In all cases, DNA extracted from the Substantia nigra of autopsy brains was used. We found seven novel missense mutations in complex I subunit genes ND1 (3992 C/T, 4024 A/G), ND4 (11253 T/C, 12084 C/T), ND5 (13711 G/A, $13768 \mathrm{~T} / \mathrm{C}$ ) and ND6 (14582 T/C). In addition, eight known missense mutations including three secondary LHON mutations (4216 T/C , 4917 A/G , 13708 G /A) as well as 43 synonymous polymorphisms, among them 20 novel sequence variants, were detected in complex I genes. Subsequent computer analysis of the novel mutations using secondary structure prediction indicated that at least one of them, a T/C transition at nucleotide position 11253 which changes a conserved isoleucine residue into threonine, is likely to contribute to PD pathogenesis. Furthermore, two new point mutations were detected in the mitochondrial tRNA genes for threonine $(15950 \mathrm{G} / \mathrm{A})$ and proline (15965 T/C). B oth sequence changes alter functionally relevant sites of the respective tRNA molecule (aminoacyl acceptor stem, TpsiC). Six known other sequence variants were detected in tRNA genes including the abovementioned $4336 \mathrm{~A} / \mathrm{G}$ mutation (Grasbon-Frodl et al., 1999).

The results of our sequencing studies indicate that the majority of all idiopathic PD cases are unlikely to be due to sequence variation in mitochondrial complex I genes. Nevertheless, mutations in mitochond rially encoded complex I and/or tRNA genes (one patient showed both) may well play a pathogenic role in a sizeable portion of PD patients, considering the high frequency of the disease. Our findings are fully in line with the observation that a complex I defect is present in between $10-30 \%$ of PD patients (A.H.V. Schapira, personal communication). In addition, mutations in nuclear complex I genes could also contribute to PD. Hattori and co-workers, for instance, have reported on a point mutation in the $24 \mathrm{kDa}$ subunit gene (NDUFV2) which appears to represent a risk factor for PD in the homozygous state (Hattori et al., 1998).

In order to combine systematic sequencing and functional analyses of mtDNA mutations, we have begun to analyze five pairs of monozygotic twins with a longitudinal diagnosis of PD using a cybrid system (Vieregge et al., 1999; Kösel et al., in preparation). Four twin pairs are presently still discordant for PD. It is of interest that five novel sequence variants were detected in the mitochond rial genes encoding complex I in four of the pairs. Two of these missense mutations affect nucleotide positions 4924 and 10192 , respectively. Both sequence variants were found in the affected as well as the unaffected twin of each pair but were absent in controls. Cybrids generated by fusion of $\rho^{0}$ cells with platelet mitochondria of the PD patients are currently being propagated in culture (Figure 2). Biochemical analyses will show whether the newly discovered mitochondrial point mutations are of functional relevance.

\section{Contribution of Mitochondrial Dysfunction to Nigral Cell Death}

It is becoming increasingly evident that one important new function of mitochondria lies in the control of cell death. In fact, mitochondria have been suggested by some authors to serve as the 'cellular centers of death control' (Hirsch et al., 1997; Lemasters et al. 1998; Susin et al., 1998). Some groups have entertained the possibility that apoptosis may be the cell death pathway underlying the neuronal demise which occurs in the parkinsonian nigra. In particular, staining results obtaind with the so-called TUNEL technique, which allows visualization of fragmentation of DNA, have been interpreted as representing signs of apoptotic cell death in melanized neurons of the Substantia nigra (M ochizuki et al., 1996; Anglade et al., 1997; Tompkins et al., 1997). Surprisingly, these changes were observed even in very late stages of the disease. Tompkins et al. (1997), in contrast, could not rule out that some necrosis had taken place in addition to apoptosis.

We have applied labeling of fragmented DNA by the TUNEL method to 22 neuropathologically verified cases of PD which had been genotyped for mtDNA mutations (Kösel et al., 1997b). Classical signs of apoptosis such as chromatin condensation in association with nuclear fragmentation and the formation of apoptotic bodies were consistenly absent from nigra neurons, whereas mild apoptosis-like changes could be observed in some glial cells. Our results are thus in line with the study of Banati and co-workers (1998) who also failed to detect evidence of apoptosis in the parkinsonian Substantia nigra. A third study had previously arrived at the same conclusion (Dragunow et al., 1995). Furthermore, PD usually develops over many years whereas apoptosis is a very rapid process. Thus, we conclude that apoptosis is not a typical autopsy finding in PD. We have proposed the new term aposklesis ('withering') to name this non-physiological mode of cell death which appears to take a slow course and which is associated with biochemical DNA but not morphological chromatin fragmentation (Graeber et al., 1999). However, heterogeneity of cell death mechanisms may be envisioned in a very common disease such as PD.

In conclusion, PD does not represent a single disease entity but comprises a genetically heterogeneous group disorders. Recent cybrid studies have shown that the complex I defect in PD is genetic and that it can arise from mtDNA. Systematic sequence analysis of the mitochondrial genome further indicates that mutations of respiratory chain complex I are present in a subset of PD patients. However, the exact mechanism which kills nigra neurons remains unknown. 


\section{References}

Anderson, S., Bankier, A.T., Barrell, B.G., de B ruijn, M.H.L., Coulson, A.R., Drouin, J., Eperon, I.C., Nierlich, D.P., Roe, B.A., Sanger, F., Schreier, P.H., Smith, A.J .H., Staden, R., and Young, I.G. (1981). Sequence organisation of the human mitochondrial genome. Nature 290, 457- 465.

Anglade, P., Vyas, S., J avoy Agid, F., Herrero, M.T., Michel, P.P., Marquez, J ., Mouatt Prigent, A., Ruberg, M., Hirsch, E.C., and Agid, Y. (1997). Apoptosis and autophagy in nigral neurons of patients with Parkinson's disease. Histol. Histopathol. 12, $25-31$.

Banati, R.B., Daniel, S.E., and Blunt, S.B. (1998). Glial pathology but absence of apoptotic nigral neurons in long-standing Parkinson's disease. Mov. Disord. 13, 221 - 227.

Benecke, R., Strümper, P., and Weiss, H. (1993). Electron transfer complexes I and IV of platelets are abnormal in Parkinson's disease but normal in Parkinson-plus syndromes. Brain 116, $1451-1463$.

Bindoff, L.A., Birch Machin, M.A., Cartlidge, N.E., Parker, W.D., J r., and Turnbull, D.M. (1991). Respiratory chain abnormalities in skeletal muscle from patients with Parkinson's disease. J . Neurol. Sci. 104, 203- 208.

Brown, M.D., Shoffner, J .M., Kim, Y.L., J um, A.S., Graham, B.H., Cabell, M.F., Gurley, D.S., and Wallace, D.C. (1996). Mitochondrial DNA sequence analysis of four Alzheimer's and Parkinson's disease patients. Am. J . Med. Genet. 61, 283- 289.

Dragunow, M., Faull, R.L.M., Lawlor, P., Beilharz, E.J ., Singleton, K., Walker, E.B., and Mee, E. (1995). In situ evidence for DNA fragmentation in Huntington's disease striatum and Alzheimer's disease temporal lobes. Neuroreport 6, 1053- 1057.

Duncan, A.M.V., Chow, W., Robinson, B.H. (1992). Localization of the human $75-\mathrm{kDal}$ Fe-S protein of $\mathrm{NADH}$-coenzyme $\mathrm{Q}$ reductase gene (NDUFS1) to $2 q 33 \rightarrow q 34$. Cytogenet. Cell. Genet. 60, $212-213$.

Egensperger, R., Kösel, S., Schnopp, N.M., Mehraein, P., and Graeber, M.B. (1997). Association of the mitochondrial tRNA(A4336G) mutation with Alzheimer's and Parkinson's diseases. Neuropathol. Appl. Neurobiol. 23, 315 - 321.

Graeber, M.B., Grasbon-Frodl, E., Abell-Aleff, P., and Kösel, S. (1999). Nigral neurons are likely to die of a mechanism other than classical apoptosis in Parkinson's disease. In: Parkinsonism and Related Disorders, in press.

Grasbon-Frodl, E.M., Kösel, S., Sprinzl, M., von Eitzen, U., Mehraein, P., and Graeber, M.B. (1999). Two novel point mutations of mitochondrial tRNA genes in histologically confirmed Parkinson disease. Neurogenetics 2, 121 - 127.

Gu, M., Cooper, J.M., Taanman, J .W., and Schapira, A.H.V. (1998). Mitochondrial DNA transmission of the mitochondrial defect in Parkinson's disease. Ann. Neurol. 44, 177- 186.

Hartley, A., Cooper, J.M., and Schapira, A.H.V. (1993). Iron induced oxidative stress and mitochondrial dysfunction: relevance to Parkinson's disease. Brain Res. 627, 349- 353.

Hattori, N., Yoshino, H., Tanaka, M., Suzuki, H., and Mizuno, Y. (1998). Genotype in the 24-kDa subunit gene (NDUFV2) of mitochondrial complex I and susceptibility to Parkinson disease. Genomics 49, 52 - 58.

Hirsch, T., Marchetti, P., Susin, S.A., Dallaporta, B., Zamzami, N., Marzo, I., Geuskens, M., and Kroemer, G. (1997). The apoptosis-necrosis paradox. Apoptogenetic proteases activated after mitochondrial permeability transition determine the mode of cell death. Oncogene 15, 1573- 1581.

Ikebe, S., Tanaka, M., and Ozawa, T. (1995). Point mutations of mitochondrial genome in Parkinson's disease. Mol. Brain Res. 28, $281-295$.
J anetzky, B., Hauck, S., Youdim, M.B.H., Riederer, P., J ellinger, K., Pantucek, F., Zöchling, R., Boissl, K.W., and Reichmann, H. (1994). Unaltered aconitase activity, but decreased complex I activity in Substantia nigra pars compacta of patients with Parkinson's disease. Neurosci. Lett. 169, $126-128$.

Kim, J.W., Lee, Y., Kang, H.B., Chose, Y.K., Chung, T.W., Chang, S.Y., Lee, K.S., Choe, I.S. (1997). Cloning of the human cDNA sequence encoding the NADH:ubiquinone oxidoreductase MLRQ subunit. Biochem. Mol. Biol. Int. 43, 669-675.

Kitada, T., Asakawa, S., Hattori, N., Matsumine, H., Yamamura, Y., Minoshima, S., Yokochi, M., Mizuno, Y., and Shimizu, N. (1998). Mutations in the parkin gene cause autosomal recessive juvenile parkinsonism. Nature 392, 605-608.

Kösel, S., Egensperger, R., Schnopp, N.M., and Graeber, M.B. (1997a). The common deletion in not increased in parkinsonian substantia nigra as shown by competitive polymerase chain reaction. Movement Disorders 12,639-645.

Kösel, S., Egensperger, R., von Eitzen, U., Mehraein, P., and Graeber, M.B. (1997b). On the question of apoptosis in the parkinsonian substantia nigra. Acta Neuropathol. 93, 105- 108.

Kösel, S., Grasbon-Frodl, E.M., Mautsch, U., Egensperger, R., von Eitzen, U., Frishman, D., Hofmann, S., Gerbitz, K.-D., Mehraein, P., and Graeber, M.B. (1998). Novel mutations of mitochondrial complex I in pathologically proven Parkinson disease. Neurogenetics 1, 197- 204.

Krige, D., Carroll, M.T., Cooper, J .M., Marsden, C.D., and Schapira, A.H. (1992). Platelet mitochondrial function in Parkinson's disease. Ann. Neurol. 32, 782 - 788.

Krüger, R., Kuhn, W., Müller, T., Woitalla, D., Graeber, M., Kösel, S., Przuntek, H., Epplen, J .T., Schols, L., and Riess, O. (1998). Ala30Pro mutation in the gene encoding alpha-synuclein in Parkinson's disease. Nature Genet. 18, 106 - 108.

Langston, J.W., Ballard, P., Tetrud, J.W., and Irwin, I. (1983). Chronic parkinsonism in humans due to a product of meperidine-analog synthesis. Anal. Biochem. 219, 979- 980.

Lemasters, J JJ ., Nieminen, A.L., Quian, T., Trost, L.C., Elmore, S.P., Nishimura, Y., Crowe, R.A., Cascio, W.E., Bradham, C.A., Brenner, D.A., and Herman, B. (1998). The mitochondrial permeability transition in cell death: a common mechanism in necrosis, apoptosis and autophagy. Biochim. Biophys. Acta 1366, 177- 196.

Leroy, E., Boyer, R., Auburger, G., Leube, B., Ulm, G., Mezey, E., Harta, G., Brownstein, M.J ., J onnalagada, S., Chernova, T., Dehejia, A., Lavedan, C., Gasser, T., Steinbach, P.J ., Wilkinson, K.D., and Polymeropoulos, M.H. (1998). The ubiquitin pathway in Parkinson's disease. Nature 395, 451- 452.

Lestienne, P., Nelson, J., Riederer, P., J ellinger, K., and Reichmann, H. (1990). Normal mitochondrial genome in brain from patients with Parkinson's disease and complex I defect. J . Neurochem. 55, 1810 - 1812.

Lücking, C.B., Abbas, N., Durr, A., Bonifati, V., Bonnet, A.M., de Broucker, T., De Michele, G., Wood, N.W., Agid, Y., and Brice, A. (1998). Homozygous deletions in parkin gene in European and North African families with autosomal recessive juvenile parkinsonism. The European Consortium on genetic susceptibility in Parkinson's Disease and the French Parkinson's Disease Genetics Study Group. Lancet 352, 1355- 1356.

Mann, V.M., Cooper, J .M., and Schapira, A.H.V. (1992). Quantitation of mitochondrial DNA deletion in Parkinson's disease. FEBS Lett. 299, 218- 222.

McNaught, K.S., Thull, U., Carrupt, P.A., Altomare, C., Cellamare, S., Carotti, A., Testa, B., J enner, P., and Marsden, C.D. (1996). Effects of isoquinoline derivates structurally related to 1methyl-4-phenyl-1,2,3,6-tetrahydropyridine (MPTP) on mitochondrial respiration. Biochem. Pharmacol. 51, 1503- 1511. 
Mizuno, Y., Ohta, S., Tanaka, M., Takamiya, S., Suzuki, K., Sato, T., Oya, H., Ozawa, T., and Kagawa, Y. (1989). Deficiencies in complex I subunits of the respiratory chain in Parkinson's disease. Biochem. Biophys. Res. Commun. 163, 1450- 1455.

Mochizuki, H., Goto, K., Mori, H., and Mizuno, Y. (1996). Histochemical detection of apoptosis in Parkinson's disease. J . Neurol. Sci. 137, $120-123$.

Ozawa, T., Tanaka, M., Ikebe, S., Ohno, K., Kondo, T., and Mizuno, Y. (1990). Quantitative determination of deleted mitochondrial DNA relative to normal DNA in parkinsonian striatum by kinetic PCR analysis. Biochem. Biophys. Res. Commun. 172, 483489.

Polymeropoulos, M.H., Lavedan, C., Leroy, E., Ide, S.E., Dehejia, A., Dutra, A., Pike, B., Root, H., Rubenstein, J ., Boyer, R., Stenroos, E.S., Chandrasekharappa, S., Athanassiadou, A., Papapetropoulos, T., J ohnson, W.G., Lazzarini, A.M., Duvoisin, R.C., Diiorio, G., Golbe, L.I., and Nussbaum, R.L. (1997). Mutation in the alpha-synuclein gene identified in families with Parkinson's disease. Science 276, 2045- 2047.

Russell, M.W., du Manoir, S., Collins, F.S., Brody, L.C. (1997). Cloning of the human NADH: ubiquinone oxidoreductase subunit B13: localization to chromosome 7q32 and identification of a pseudogene on 11p15. Mamm. Genome 8, 60-61.

Schapira, A.H.V., Cooper, J .M., Dexter, D., J enner, P., Clark, J .B., and Marsden, C.D. (1989). Mitochondrial complex I deficiency in Parkinson's disease. Lancet 1, 1269.

Schapira, A.H.V., Mann, V.M., Cooper, J .M., Dexter, D., Daniel, S.E., J enner, P., Clark, J .B., and Marsden, C.D. (1990). Anatomic and disease specificity of NADH COQ 1 reductase (complex I) deficiency in Parkinson's disease. J. Neurochem. 55, 2142 2145.

Shoffner, J .M., Brown, M.D., Torrini, A., Lott, M.T., Cabell, M.F., Mirra, S.S., Beal, M.F., Yang, C.-C., Gearing, M., Salvo, R., Watts, R.L., J uncos, J .L., Hansen, L.A., Crain, B.J ., Fayed, M.,
Reckord, C.L., and Wallace, D.C. (1993). Mitochondrial DNA variants observed in Alzheimer disease and Parkinson disease patients. Genomics 17, 171- 184.

Sian, J ., Dexter, D.T., Lees, A.J ., Daniel, S., Agid, Y., J avoy Agid, F., $J$ enner, P., and Marsden, C.D. (1994). Alterations in glutathione levels in Parkinson's disease and other neurodegenerative disorders affecting basal ganglia. Ann. Neurol. 36, 348 - 355.

Susin, S.A., Zamzami, N., and Kroemer, G. (1998). Mitochondria as regulators of apoptosis: doubt no more. Biochim. Biophys. Acta 1366, $151-165$.

Swerdlow, R.H., Parks, J.K., Miller, S.W., Tuttle, J.B., Trimmer, P.A., Sheehan, J .P., Bennett, J .P., Davis, R.E., and Parker, W.D. (1996). Origin and functional consequences of the complex I defect in Parkinson's disease. Ann. Neurol. 40, 663-671.

Swerdlow, R.H., Parks, J .K., Davis, J .N., Cassarino, D.S., Trimmer, P.A., Currie, L.J ., Dougherty, J ., Bridges, W.S., Bennett, J .P., Wooten, G.F., and Parker, W.D. (1998). Matrilineal inheritance of complex I dysfunction in a multigenerational Parkinson's disease family. Ann. Neurol. 44, 873-881.

Tabrizi, S.J ., Cooper, J.M., and Schapira, A.H.V. (1998). Mitochondrial-DNA in focal dystonia - a cybrid analysis. Ann. Neurol. 44, $258-261$.

Tompkins, M.M., Basgall, E.J ., Zamrini, E., and Hill, W.D. (1997). Apoptotic-like changes in Lewy-body-associated disorders and normal aging in substantia nigra neurons. Am. J. Pathol. $150,119-131$.

Vieregge, P., Hagenah, J ., Heberlein, I., Klein, C., and Ludin, H.-P. (1999). Parkinson disease in twins: A follow-up study. Neurology, in press.

Wallace, D.C. (1992). Diseases of the mitochondrial DNA. Annu. Rev. Biochem. 61, 1175- 1212 .

Wooten, G.F., Currie, L.J ., Bennett, J .P., Harrison, M.B., Trugman, J .M., and Parker, W.D. (1997). Maternal inheritance in Parkinson's disease. Ann. Neurol. 41, 265- 268. 